\title{
Absence de restes dans une société non occidentale
}

Les Ari du Sud-Ouest de l'Éthiopie

Non-Waste in a Non-Western Society: The Aari of Southwestern Ethiopia

\section{Morie Kaneko}

\section{(2) OpenEdition}

Journals

Édition électronique

URL : https://journals.openedition.org/tc/7839

DOI : $10.4000 /$ tc. 7839

ISSN : 1952-420X

Éditeur

Éditions de l'EHESS

Édition imprimée

Date de publication : 31 octobre 2016

Pagination : 134-137

ISBN : 9782713225291

ISSN : 0248-6016

Référence électronique

Morie Kaneko, "Absence de restes dans une société non occidentale», Techniques \& Culture [En ligne], 65-66 | 2016, mis en ligne le 31 octobre 2016, consulté le 29 septembre 2022. URL : http://

journals.openedition.org/tc/7839; DOI : https://doi.org/10.4000/tc.7839 


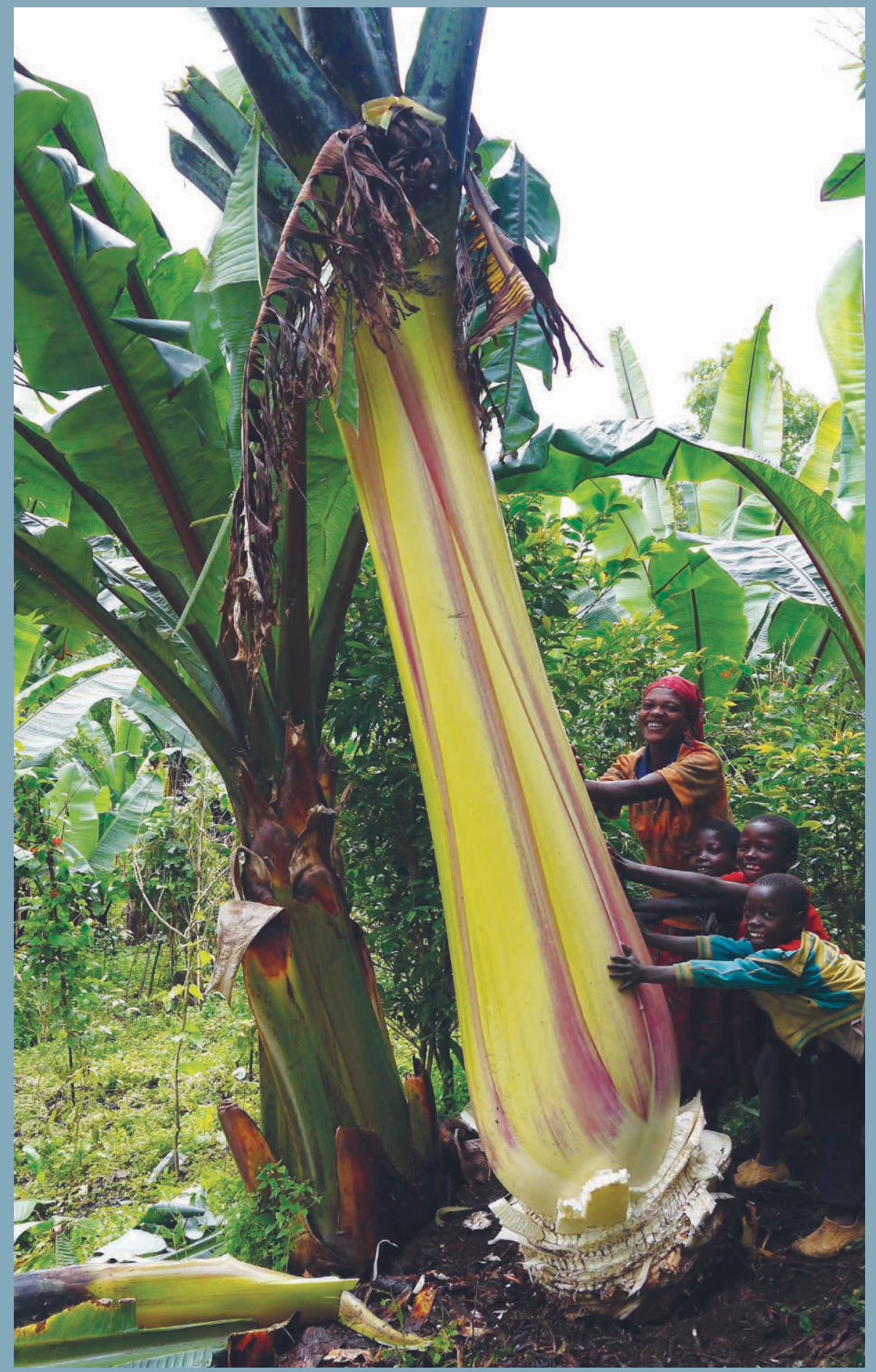




\section{Absence de restes}

dans une société non occidentale

\section{Les Ari du Sud-Ouest de l’Éthiopie}

Sur mon terrain, le Sud-Ouest de l'Éthiopie, il est courant de trouver des restes de plantes, et des sacs en plastique dans les endroits les plus fréquentés, tels que les marchés et les chemins. Mais il est rare que les gens les désignent par des termes collectifs tels que « déchets». J’ai assisté à deux scènes ayant comme objet central ces détritus. La première se rapportait à une femme travaillant au marché, assise à côté de gaines foliaires séchées. Elle n'en n'avait plus l'usage mais ne les qualifiait pas pour autant de déchets. La seconde correspond à des cahiers d'écoliers relégués et entassés sur une planche de bois à côté de fragments de papiers éparpillés sur le sol. Ceux-ci n'étaient pas non plus considérés comme des déchets. Ces simples impressions montrent qu'en langue Ari, les catégories linguistiques sont en décalage avec ce que nous désignons (en Orient ou en Occident) en termes d'utilité.

D’après mon informateur - un homme âgé d'une cinquantaine d'années ayant reçu une éducation moderne et travaillant pour une ONG - les Ari utilisent le terme koshasha, emprunté de l'amharique, pour désigner les déchets.

Le sujet de cet article tient compte d'au moins deux dimensions liées à l'attitude des individus dans le traitement des vieux cahiers d'écolier. L'une concerne la manière dont les habitants se débarrassent des choses dans leur vie quotidienne, l'autre touche à leur rapport à l'archivage de documents destinés à conserver les connaissances et les faits. Afin de comprendre le phénomène auquel j'ai été confrontée dans cette région, il est important d'analyser ces deux questions.

Les deux éléments sur lesquels je me concentre d'abord ici sont l'ensète [NdT : plante également appelée «bananier d'Abyssinie »] et la poterie, qui ne sont jamais considérés comme koshasha. L'une des raisons en est qu'ils demeurent au sein de la circulation matérielle. Celle-ci est encouragée par les pratiques agricoles et artisanales du peuple Ari. Leurs pratiques se basent sur leurs activités quotidiennes découlant des expériences d'interaction qu'ils 


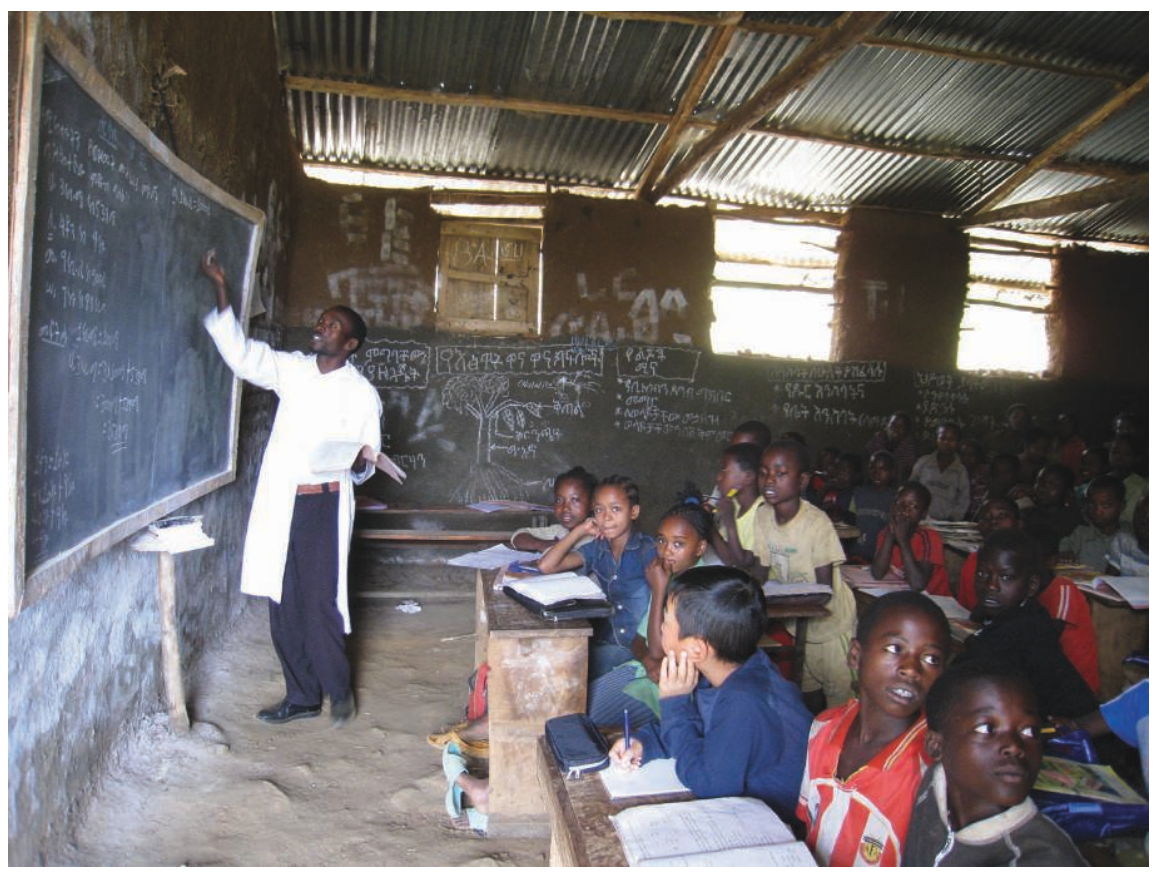

- Si les cahiers sont une ressource indispensable aux écoliers, leur façon de les conserver diffère en fonction de la classe d'âge et du niveau de scolarisation. entretiennent avec leur environnement. Cet article observe les objets comme un prolongement des mouvements du corps et les corps comme des extensions d'objets, ce qui souligne l'affordance générée par la relation entre les deux phénomènes. Partant de cette approche, nous considérons que les comportements coopératifs et les pratiques culturelles naissent des interactions entre les corps et leur environnement.

Le concept de déchets chez les Ari du Sud-Ouest de l'Éthiopie est abordé en décrivant le cycle de vie de l'ensète qui sert de nourriture de base, celui des poteries en tant qu'ustensiles de cuisine et enfin celui des cahiers d'écoliers. L'analyse procède de données relatives à la production et la consommation d'ensète, recueillies en juillet 2012 et janvier 2014, au processus d'utilisation des poteries, recueillies pendant environ un an et demi entre 1999 et 2001, et enfin d'entretiens menés auprès de 47 étudiants en mars 2013.

En règle générale, les poteries ainsi que les artéfacts à base d'ensète circulent au sein de l'écosystème sans jamais devenir des déchets. Les Ari les utilisent et les réinsèrent dans le système en faisant appel à leurs connaissances et à leurs techniques, intégrées à l'environnement socioécologique. Leur rapport aux vieux cahiers d'école élémentaire est semblable à celui qu'ils ont aux connaissances et aux techniques locales. Par contraste, rappelons que les collégiens et les lycéens débutent leur apprentissage des savoirs modernes et des techniques par l'écriture des connaissances, en accumulant des notes sur le papier puis en les mémorisant.

L'utilisation de l'ensète comme la pratique de la poterie sont soumises à une évaluation par l'expérience du corps. Le terme « connaissance » est exprimé par le mot eskan, qui désigne une forme de savoir fondé sur l'expérience, c'est le cas dans la production d'ensète ou l'utilisation des poteries. À l'inverse, le système éducatif des écoles modernes repose sur l'apprentissage de connaissances partant de faits scientifiques. L'activation des concepts de dakari (mauvais) et de wanna (bon) peut présenter quelques difficultés pour les étudiants une fois qu'ils sont habitués à appréhender la connaissance comme un ensemble d'informations ne reposant pas sur l'expérience. L'ensète et les poteries sont considérées comme dakari en fonction d'un degré d'utilité dont l'échelle est progressive ou continue. 
L'enseignement scolaire est délivré par les professeurs aux élèves. Les cahiers sont un support important pour ces derniers. Parce que le savoir scolaire ne fait pas de distinction, en langue ari, entre eskan, «savoir» et shedinkan, qui signifie «voir», ces connaissances sont appréciées à l'aune de leur nécessité/non-nécessité. Compte tenu de ces considérations, il n'existe pas de notion de déchets chez les Ari qui dépendrait de l'opposition entre nécessaire et non nécessaire. Les études de cas sur la mise en réserve des cahiers usagés ou leur stockage dans des sacs de café, montrent une interpénétration entre le concept de dakari entendu selon le critère de l'utilité et le concept de dakari entendu selon celui de la nécessité.

\section{I'auteure}

Morie Kaneko, docteure en Études régionales, est professeure agrégée à l'École Supérieure des Études Asiatiques et Africaines (ASAFAS - Graduate School of Asian and African Area Studies) de l'université de Kyoto, au Japon. Ses recherches ethnographiques portent sur la transmission culturelle chez les artisans d'Éthiopie. L'une de ses récentes publications s'intitule Gender-based Knowledge and Techniques in Africa (2013).

\section{Iconographie}

Image d'ouverture. L'ensète (ensete ventricosum) est une plante originaire d'Éthiopie. Elle est cultivée uniquement dans le Sud de l'Éthiopie. Sa hauteur maximale est d'environ 5 mètres et sa récolte se fait tout au long

de l'année. Sa culture procure à plus de 20 millions de personnes en Éthiopie, la principale nourriture de base. (C) Morie Kaneko.

1. (C) Morie Kaneko.

\section{Pour citer cet article}

Kaneko, E. 2016 «Absence de restes dans une société non occidentale. Les Ari du Sud-Ouest de l'Éthiopie», TechniquesECulture 65-66 «Réparer le monde. Excès, reste et innovation », p. 134-137. 